\title{
Electro-mechanical modeling of dielectric elastomer transducers with micro-structured electrodes
}

\author{
A. Schmidt ${ }^{* a, b}$, A. Bergamini ${ }^{\mathrm{b}}$, C. Jordi ${ }^{\mathrm{b}}$, G. Kovacs ${ }^{\mathrm{b}}$, E. Mazza $^{\mathrm{a}, \mathrm{b}}$ \\ ${ }^{a}$ Swiss Federal Institute of Technology ETH, Department of Mechanical and Process Engineering \\ Tannenstrasse 3, 8092 Zurich, Switzerland; $\quad{ }^{b}$ Swiss Federal Laboratories for Materials Science \\ and Technology Empa, Ueberlandstrasse 129, 8600 Duebendorf, Switzerland
}

\begin{abstract}
Recently micro-structured solid electrodes were applied to dielectric elastomers. Compared with common powder or liquid electrodes the electrical conductivity of the electrodes is enhanced while the compliance necessary for large active deformations is retained. Envisaged applications range from energy harvesting to structural damping and actuation. The compliant conducting electrodes can be attached to the passive dielectric materials in a standardized and well controlled process. This enhanced the general interest in the technology and more applications are expected. While some of these applications aim for maximum actuation performance others require a superior reliability at moderate performance. For both strategies design and optimization of the active parts are essential. This work provides results of an extensive experimental characterization of the passive and active response of a novel PolyPower membrane. We further developed a 3D nonlinear viscoelastic model suitable for finite element simulation and verified the main assumptions of the modeling approach with mechanical tests. The model is shown to provide good predictions of both passive behavior as well as active deformation of an actuator system.
\end{abstract}

Keywords: dielectric, corrugated, modeling, hyperelastic, PolyPower, DEAP, isotonic, relaxation

\section{INTRODUCTION}

Dielectric elastomers are soft insulating materials that have attracted scientific interest for their ability to convert electrical into mechanical energy and vice versa. By applying an electric field onto an insulating material, the field will lead the material to deform dependent on its specific material properties. For very soft dielectrics, electric fields well below the materials breakdown strength causes noticeable active deformations or if deformations are constraint active forces arise that are used in dielectric elastomer actuators (DEA) ${ }^{1-5}$. More recently the applicability of dielectric elastomers for electrical energy harvesting was investigated ${ }^{6-9}$. Lately a structure of a corrugated silicone membrane coated on opposite sides with conducting silver became available ${ }^{10}$. This PolyPower DEAP (by Danfoss) can be elongated in one direction (Figure 1) without breaking the solid silver coating. Due to the corrugation of the thin structure (about $0.2 \mu \mathrm{m}$ thickness and $5 \mu \mathrm{m}$ height and period ${ }^{11}$ ) the strains locally present on the silver coating are very small despite large deformations applied to the membrane. The corrugation is applied to the silicon before cross-linking therefore no significant internal pre-stress is expected in the structure.

Electroactive Polymer Actuators and Devices (EAPAD) 2011, edited by Yoseph Bar-Cohen, Federico Carpi, Proc. of SPIE Vol. 7976, 79760L · C 2011 SPIE · CCC code: 0277-786X/11/\$18 · doi: 10.1117/12.880287 
Since electrode and passive dielectric are fabricated in an industrial process, cheap and easily available membranes readily prepared for actuator fabrication are now available and an increasing number of applications is currently being developed ${ }^{10,12}$. While some applications require high actuation forces and large deformations others necessitate a superior long-term reliability at moderate performance. All in common the development of successful applications mostly requires modeling for design and optimization of the device. This work provides material model parameter that can be used to simulate the nonlinear time dependent behavior with commercially available finite element (FE) software. We concluded a large number of passive mechanical tests to characterize the material behavior as well as to verify key-assumptions necessary for the model development. Parameters of a hyper-viscoelastic model formulation are provided. Further the material is characterized in a standard actuator experiment. These so called "isotonic activation" tests were chosen since a simple analytical solution can be derived.

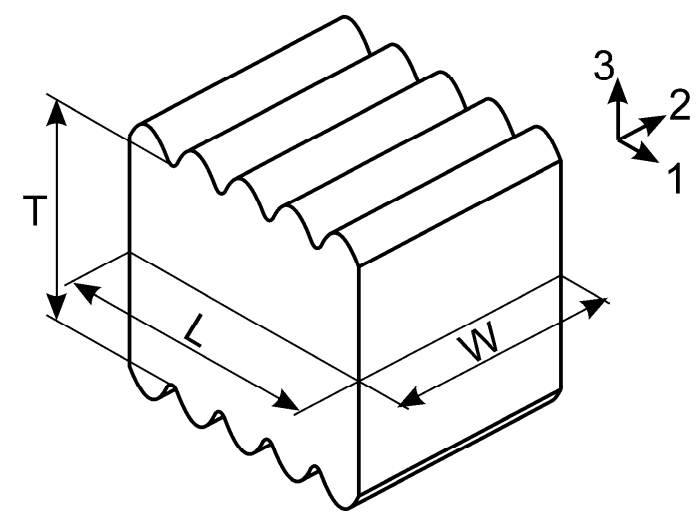

Figure 1 PolyPower membrane with micro-structured surface

\section{Experiments}

All experiments were carried out at $22^{\circ} \mathrm{C}$ and $50 \%$ relative humidity in a laboratory at the Institute of Mechanical Systems IMES at ETH Zurich. Samples were kept in the lab for more than $24 \mathrm{~h}$ before testing.

\subsection{TENSILE TESTS}

To characterize the passive mechanical response of PolyPower DEAP tensile tests in direction 1 (see Figure 1) were conducted. Tensile tests were carried out using a hydraulic tensile testing device (MTS) with a $1 \mathrm{~N}$ or for the larger samples a $20 \mathrm{~N}$ force gauge. All samples of the tensile tests were die cut. Three groups of samples characterized by a different aspect ratio of length to width were tested. The dimension of the different sample shapes are reported in Table 1. Further samples of shape A were tested with different nominal strain rates from $0.0067 \mathrm{~s}^{-1}$ to $10 \mathrm{~s}^{-1}$. See Table 2 for an overview of all experiments.

To calculate stress values from measured force signals a thickness of $\mathrm{T}=0.09 \mathrm{~mm}$ (Figure 1), as derived from optical microscopy ${ }^{13}$ is used. Results of Cauchy-stress (calculated assuming incompressibility) in direction 1 calculated as average of 5 measurements are given in Figure 2 (a) for each sample shape tested with a strain rate of $0.0067 \mathrm{~s}^{-1}$. As can be seen all samples reveal a 
nonlinear behavior. Error bars indicate the observed standard deviation of less than $4 \%$, thus demonstrating high measurement accuracy as well as very reproducible material behavior. For samples with a small aspect ratio of length to width (shape B and C) the constraint transverse deformation at the clamping can lead to a nonzero stress component in direction 2 if the samples are elongated in direction 1. For an isotropic material a higher stiffness is therefore expected for these samples. However, in Figure 2 (a) can be seen that the aspect ratio of length to width has apparently only a minor effect on the stiffness. We attribute this to the pronounced anisotropy of the membrane related to the high transverse stiffness of the corrugated electrodes that leads to a state of two dimensional deformation in all samples. We attribute the somewhat smaller stiffness of the samples $\mathrm{C}$ at large deformations to some slipping at the clamping.

Figure 2 (b) reports the average Cauchy-stress calculated for the experiments with different strain rates (sample shape A). As expected from a silicone elastomer, the difference of these four curves is rather small. Note that especially the results obtained with a strain rate of 0.033 and $0.0067 \mathrm{~s}^{-1}$ are almost identical: these measured stress-strain relations are close to the long-term material response.

Table 1 Sample dimensions

\begin{tabular}{ccc}
\hline Shape & length L [mm] & width W [mm] \\
\hline A & 25 & 4 \\
\hline B & 52 & 200 \\
\hline C & 20 & 200 \\
\hline Actuators & 37 & 200 \\
\hline
\end{tabular}

Table 2 Test matrix

\begin{tabular}{|c|c|c|c|c|c|c|c|c|c|c|c|}
\hline \multirow{3}{*}{ Shape } & \multicolumn{4}{|c|}{ Tensile } & \multicolumn{3}{c|}{ Isotonic } & \multicolumn{3}{c|}{ Relaxation } & \multirow{2}{*}{ Cyclic tensile } \\
\cline { 2 - 12 } & \multicolumn{4}{|c|}{ Strain rate $\left[\mathrm{s}^{-1}\right]$} & \multicolumn{3}{c|}{ Voltage [kV] } & \multicolumn{3}{c|}{ Max. strain [\%] } & \multirow{2}{*}{} \\
\cline { 2 - 12 } & 0.0067 & 0.033 & 0.33 & 10 & 0 & 2 & 3 & 50 & 75 & 100 & \\
\hline A & 5 & 5 & 5 & 6 & - & - & - & 5 & 7 & 6 & 6 \\
\hline B & 3 & 3 & 3 & - & - & - & - & - & - & - & - \\
\hline C & 3 & - & - & - & - & - & - & - & - & - & - \\
\hline Actuators & - & - & - & - & 3 & 3 & 3 & - & - & - & - \\
\hline
\end{tabular}




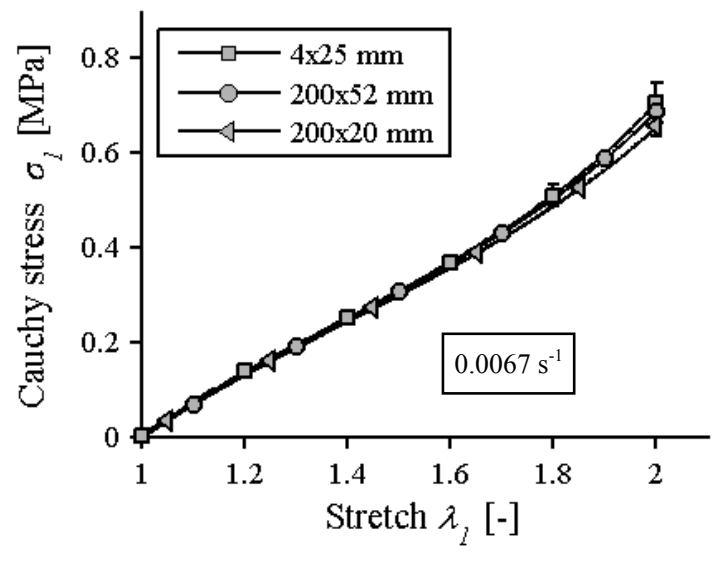

(a)

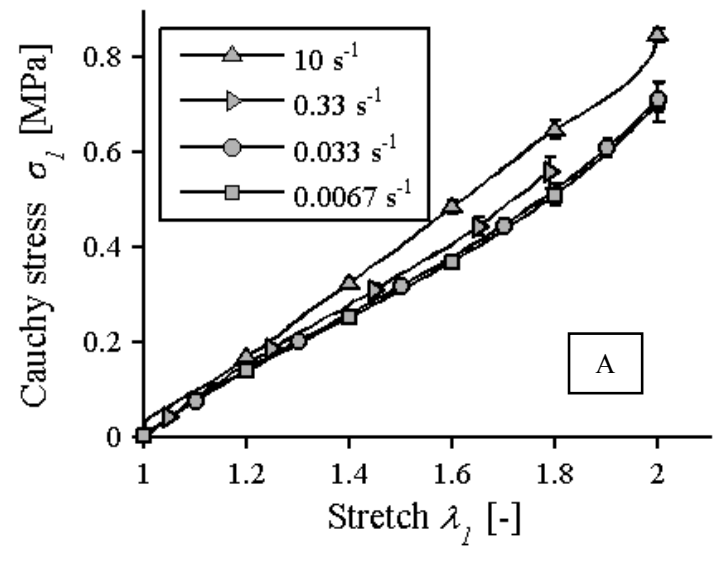

(b)

Figure 2 Cauchy stress in tensile tests with a) samples of different aspect ratio and b) different strain rates (sample shape A)

\subsection{RELAXATION TESTS}

Relaxation tests with strips of sample shape A (Table 1) were conducted to characterize the timedependence of passive mechanical response of PolyPower DEAP. After a deformation ramp with a strain rate of $10 \mathrm{~s}^{-1}$ the material response was measured for more than $1800 \mathrm{~s}$ at a constant nominal strain of 50,75 or $100 \%$. The average of five to seven samples of each strain value as well as the observed standard deviation are reported in Figure 3. The amount of stress reduction is in the range of $20 \%$ and almost unaffected by the value of strain employed. The corresponding variability is less than $5 \%$. It should be noted that relaxation continues at low rates after the observation period of $1800 \mathrm{~s}$.

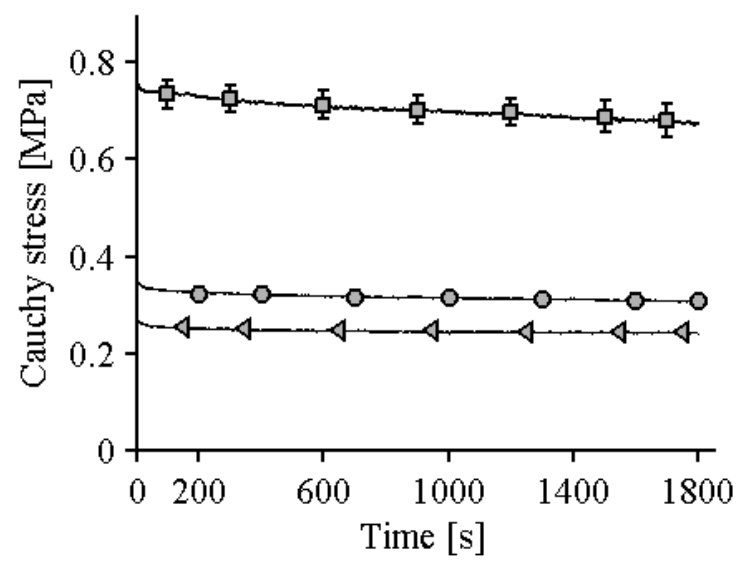

(a)

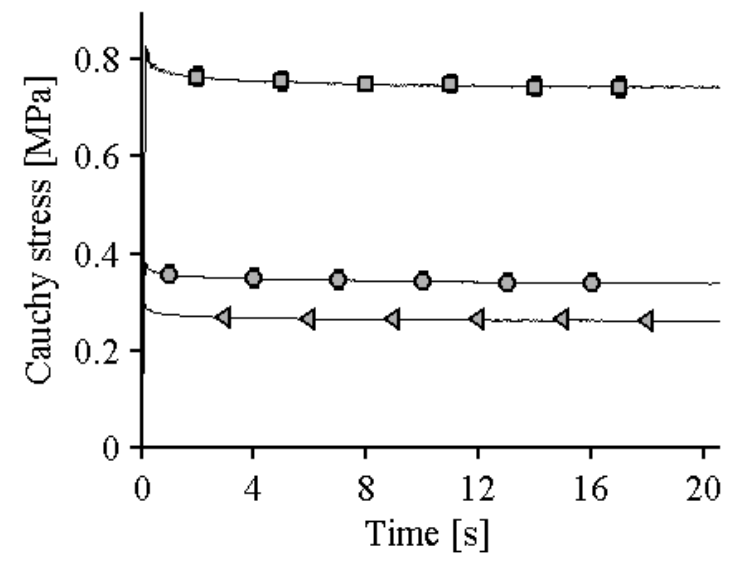

(b)

Figure 3 Cauchy stress in relaxation experiments a) for the whole time range b) detailed plot up to $20 \mathrm{~s}$, (square symbols $100 \%$ strain, circles $75 \%$ and triangles $50 \%$, error bars indicate the observed standard deviation) 


\subsection{CYCLIC TENSILE TESTS}

Cyclic tensile tests were conducted on the same set-up used for the tensile and relaxation tests. Within $10 \mathrm{~s}$ an initial deformation of $40 \%$ is applied onto samples of shape A. After a dwell time of $300 \mathrm{~s}$, a sinusoidal deformation with amplitude of $\pm 10 \%$ (relative to the undeformed sample length) and a frequency of $1 \mathrm{~Hz}$ is applied. Deformation as well as tensile force is recorded for 30 cycles. Subsequently a second deformation ramp up to $80 \%$ within $10 \mathrm{~s}$ followed by a relaxation hold of $300 \mathrm{~s}$ is applied. At this deformations a second cyclic deformation with amplitude $\pm 2 \%$ and frequency of $5 \mathrm{~Hz}$ is applied for 30 cycles. Figure 4 shows the displacement control profile of the cyclic tensile tests. In Figure 5 the average of measured stretch and Cauchy-stress of six samples is given. To enhance the visibility data is plotted for the first four to five cycles of each frequency and the force signal is smoothed using a moving average with a span width of 10 data points. As can be seen there is only a very small phase lag between stress and stretch $(<0.1 \%$ of period $)$.

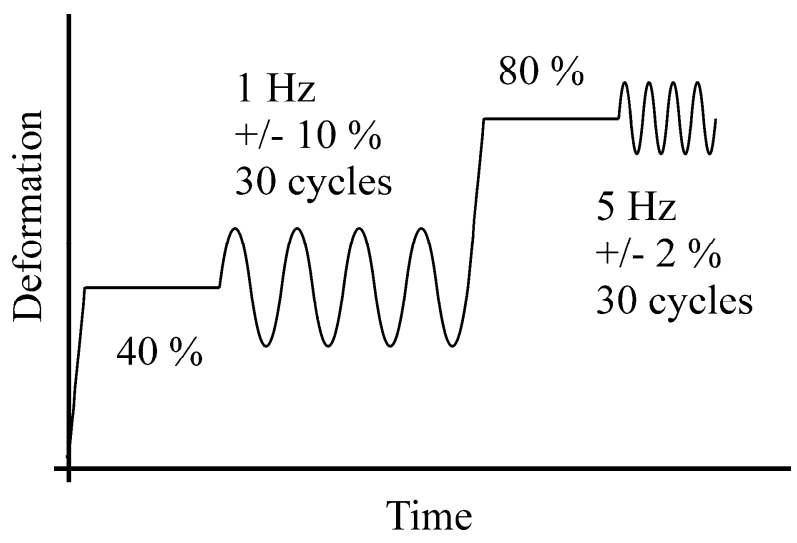

Figure 4 Displacement control profile of cyclic tensile tests

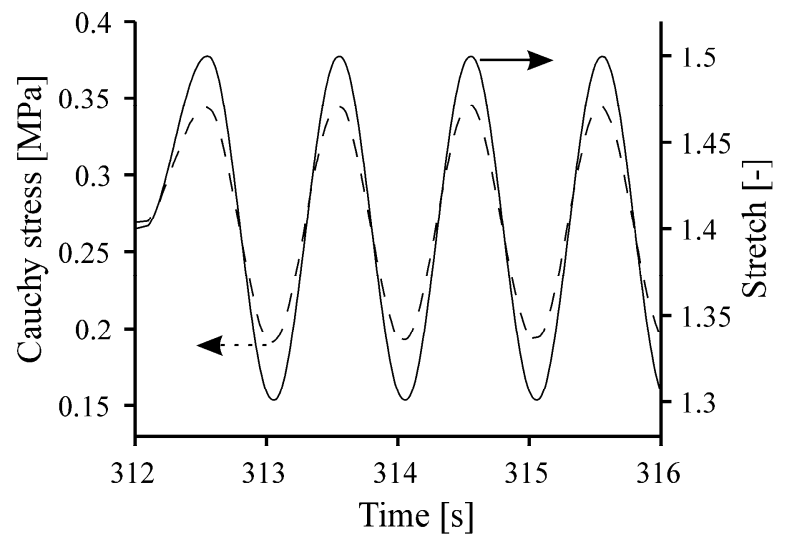

(a)

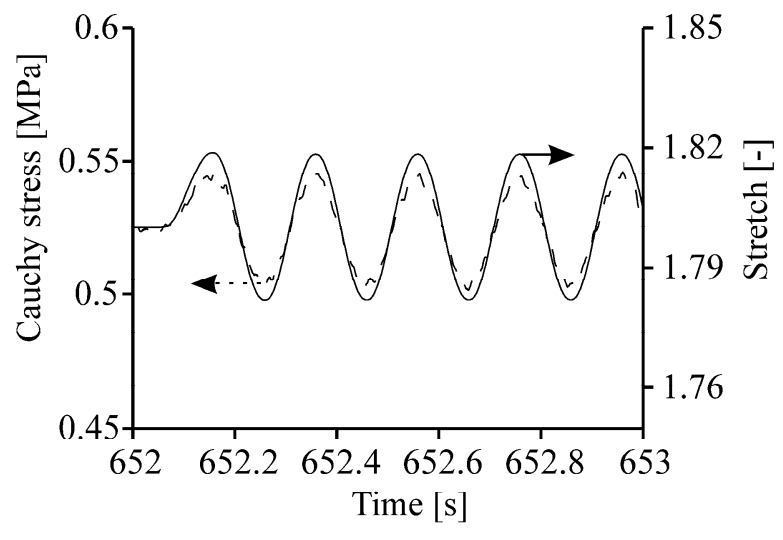

(b)

Figure 5 Stretch and Cauchy stress in cyclic tensile tests, (a) for strain amplitude of $\pm 10 \%$ and a frequency of $1 \mathrm{~Hz}$, (b) for a strain amplitude of $\pm 2 \%$ and a frequency of $5 \mathrm{~Hz}$ 


\subsection{ISOTONIC ACTIVATION TESTS}

To complement the data from mechanical tests, electro-mechanical experiments with PolyPower DEAP are presented. Specifically isotonic activation tests were performed to characterize the active deformation at different levels of stress and electric field. Samples were prepared by cutting the membranes to a rectangular shape of dimensions $200 * 37 \mathrm{~mm}$. The corrugated silver electrode was removed with a clorine solution from $10 \mathrm{~mm}$ from the edges to ensure electric isolation at high electric fields. With thin strips of aluminum foil each of the electrodes were connected to a high voltage power supply (Trek Inc. Model 5/80). With the help of PTFE frames the samples were clamped and loaded with passive weights ranging from $0.03 \ldots 0.7 \mathrm{~kg}$. The elongation caused by the passive weight was measured using an optical measurement set-up. From elongation and passive weight values of principle stretch and Cauchy-stress are calculated. The values shown in Figure 6 correspond to recorded values of elongation $30 \mathrm{~s}$ after the application of the weight. As can be seen for a given weight the stretch increases with the applied voltage. This is commonly referred to as "active deformation" and calculated as a strain relative to the deformation state at zero electric field. Values of active deformation are in the range of $0.6 \ldots 3.2 \%$ for $2 \mathrm{kV}$ and $1.6 \ldots 7.7 \%$ for $3 \mathrm{kV}$.

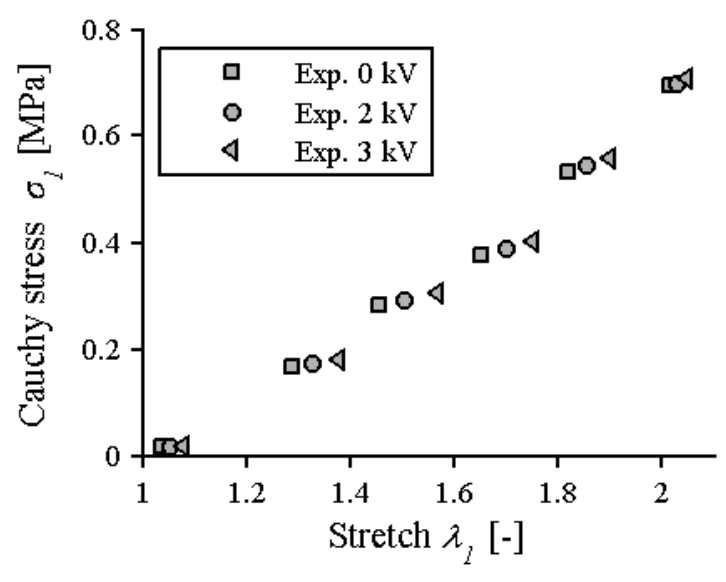

Figure 6 Cauchy stress vs. stretch in isotonic activation for different voltage levels

\section{Modeling}

With the data reported in section 2 we derive a nonlinear time-dependent constitutive model suitable for finite element simulation. Specifically a hyperelastic Yeoh-model formulation is chosen. This model-formulation was shown to accurately predict the behavior of different nonlinear elastic materials $^{14-16}$ and is readily implemented in most commercial $F E$ software. We further investigated the predictive capabilities of an Arruda-Boyce and an Ogden-model formulation for the PolyPower DEAP and found them to not perform as well as the presented Yeoh-model. The influence of the electrodes will be represented by a kinematic constraint (see section 3.1). Since the relaxation behavior of PolyPower DEAP is independent of the imposed value of strain (Figure 8), a quasi-linear viscoelastic model is used for the dissipative part of the mechanical material behavior. With this approach the elastic and dissipative part of the constitutive model can be determined separately. Data 
of tensile tests (section 2.1) are used to derive parameters of the elastic models. The viscoelastic model is fitted to results of relaxation tests (section 2.2) and will be formulated in both, time and frequency domain.

\subsection{HYPERELASTIC MODEL}

The nonlinear hyperelastic Yeoh-model ${ }^{17}$ expresses the strain energy density $W$ stored in the material as a function of the first invariant $I_{l}$ of the Right Cauchy-Green deformation tensor $\underline{C}$ :

$$
W=C_{1}\left(I_{1}-3\right)+C_{2}\left(I_{1}-3\right)^{2}+C_{3}\left(I_{1}-3\right)^{3}
$$

with:

$$
I_{1}=\lambda_{1}^{2}+\lambda_{2}^{2}+\lambda_{3}^{2}
$$

The pronounced stiffness of the corrugated electrode in direction 2 prevents almost any deformation in this direction. Thus a kinematic constraint is introduced with the stretch in the transverse direction $\lambda_{2}=1$. Assuming the membrane to be incompressible $\left(\lambda_{1} \lambda_{2} \lambda_{3}=1\right)$ the state of deformation in the PolyPower DEAP membrane can be expressed by the stretch in the compliant direction $\lambda_{I}$ since $\lambda_{3}=1 / \lambda_{1}$. Therefore:

$$
I_{1}=\lambda_{1}^{2}+1+\frac{1}{\lambda_{1}^{2}}
$$

For a hyperelastic material the Cauchy-stress tensor $\underline{\sigma}$ can be calculated by taking the derivative of $W$ with respect to the Right Cauchy-Green deformation tensor $\underline{C}^{18}$ :

$$
\underline{\sigma}=2 \underline{F} \frac{\partial W}{\partial \underline{C}} \underline{F}^{T}-p \underline{I}=2 \underline{F} \frac{\partial W}{\partial I_{1}} \frac{\partial I_{1}}{\partial \underline{F}} \underline{F}^{T}-p \underline{I}=2 \underline{F} \frac{\partial W}{\partial I_{1}} \underline{I}^{T}-p \underline{I}
$$

with unit tensor $\underline{I}$, hydrostatic pressure $p$ and deformation gradient $\underline{F}$ for the specific situation of the PolyPower DEAP:

$$
\underline{F}=\left[\begin{array}{ccc}
\lambda_{1} & 0 & 0 \\
0 & 1 & 0 \\
0 & 0 & 1 / \lambda_{1}
\end{array}\right]
$$

For the tensile tests described in section 2.1 the hydrostatic pressure $p$ can be identified from the fact that the stress in direction 3 equals zero:

$$
p=2\left(\lambda_{3}^{2} \frac{\partial W}{\partial I_{1}}\right)
$$

and thus inserting (1), (3) and (6) into (4) the Cauchy stress in direction 1 can be calculated. Predictions of the Yeoh-model are compared with experimental data of tensile tests. By iteratively adapting the material parameters $C_{j}$, eq. (4) is fitted to the experimental data obtained at low rate of deformation, thus leading to the so called "long-term" elastic parameters. All determined material 
parameters are reported in Table 3. Figure 7 shows the measured and calculated stress-strain relation according to eq. (4). As can be seen the model describes the material behavior very well.

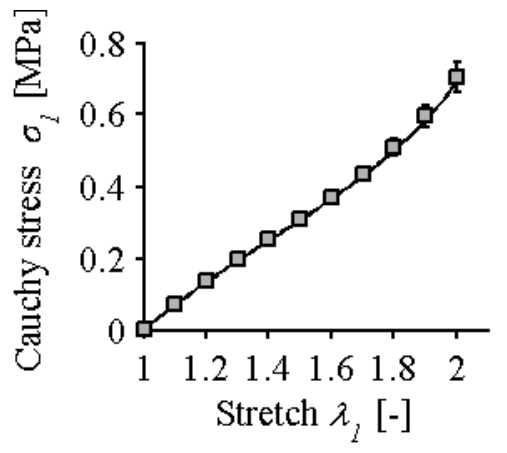

Figure 7 Cauchy stress vs. stretch for a strain rate of $0.0067 \mathrm{~s}^{-1}$ experimental data (square symbols) and predictions of Yeoh-model formulation (line)

\subsection{VISCOELASTIC MODEL}

The data of Cauchy stress from relaxation tests at different values of strain (Figure 3) are normalized with respect to the corresponding peak stress. The normalized stresses are shown in Figure 8. As can be seen the relaxation behavior of the PolyPower DEAP membrane is almost independent of the imposed level of strain. We therefore assume the material to obey a quasi-linear viscoelastic behavior. Thus the time-dependence can be expressed with time-dependent coefficients $C_{j}$, of the hyperelastic model (eq. (a)). The so called "Prony"-series express the coefficients $C_{j}$ as, ${ }^{19}$ :

$$
C_{j}=C_{j 0}\left(1-\sum_{k}^{n} g_{k}\left(1-e^{\left(-t / t_{k}\right)}\right)\right)
$$

with $C_{j 0}$ the coefficients describing the instantaneous response and the characteristic relaxation times $t_{k}$ with their respective weighting factors $g_{k}$.

The stress-strain relations from the tensile tests with strain rates of $0.033 \mathrm{~s}^{-1}$ and $0.0067 \mathrm{~s}^{-1}$ almost coincide (Figure 2). Further the long-term stress values from relaxation experiments at different stretch levels are very close to the respective values from monotonic tensile tests with a strain rate $0.0067 \mathrm{~s}^{-1}$, compare Figure 2 and 3 . For this reason we consider the hyperelastic coefficients determined from data of a strain rate of $0.0067 \mathrm{~s}^{-1}$ the long-term parameters $C_{j \infty 0}$. The coefficients of instantaneous response $C_{j 0}$ can be calculated by:

$$
C_{j 0}=C_{j \infty} /\left(1-\sum_{k}^{N} g_{k}\right)
$$

In order to derive values of viscoelastic material parameters $g_{k}$ and $t_{k}$, the dimensionless relaxation function $g(t)$ :

$$
g(t)=1-\sum_{k}^{4} g_{k}\left(1-e^{\left(-t / t_{k}\right)}\right)
$$

is fitted to the normalized stress values of the relaxation experiments (Figure 8). 
All viscoelastic material parameters are reported in Table 3. A comparison of experimental data and model predictions according to eq. (9) is given in Figure 9. The quasi-linear viscoelastic model accurately describes the experimental data. Note that, since no experimental data is available for very high strain rates (above $10 \mathrm{~s}^{-1}$ ) and very long dwell times (above $1800 \mathrm{~s}$ ), the predictive capabilities of the model outside this range cannot be evaluated.

A variety of applications uses the PolyPower DEAP membrane in cyclic loading configurations ${ }^{20}$. The mechanical response will be characterized by a phase shift between deformation and force. Such a behavior can be described with the complex formulation (loss- and storage modulus). Similar the coefficients of the nonlinear elastic material model are expressed in complex form:

$$
C_{i}(\omega)=C_{i R}(\omega)+i C_{i I}(\omega)
$$

The values of storage and loss fraction $C_{i R}$ and $C_{i I}$ of the hyperelastic coefficients can readily be determined from the Laplace transformation of the time-dependent model (eq. (7)):

$$
C_{i}(\omega)=C_{i 0}\left(1-\sum_{k=1}^{4} g_{k}\left(1-\frac{\omega^{2} t_{k}}{1+\omega^{2} t_{k}^{2}}\right)\right)+i C_{i 0}\left(\sum_{k=1}^{4} g_{k}\left(\frac{\omega t_{k}}{1+\omega^{2} t_{k}^{2}}\right)\right)
$$

Predictions of the time- (or frequency) dependent model are compared with the experimental data of cyclic tensile tests in section 3.3.

Table 3. Constitutive model parameters

\begin{tabular}{|c|c|c|c|c|c|c|c|c|c|c|}
\hline $\begin{array}{c}C_{1} \\
{[\mathrm{MPa}]}\end{array}$ & $\begin{array}{c}C_{2} \\
{[\mathrm{MPa}]}\end{array}$ & $\begin{array}{c}C_{3} \\
{[\mathrm{MPa}]}\end{array}$ & $\mathrm{g}_{1}[-]$ & $\mathrm{t}_{1}[\mathrm{~s}]$ & $\mathrm{g}_{2}[-]$ & $\mathrm{t}_{2}[\mathrm{~s}]$ & $\mathrm{g}_{3}[-]$ & $\mathrm{t}_{3}[\mathrm{~s}]$ & $\mathrm{g}_{4}[-]$ & $\mathrm{t}_{4}[\mathrm{~s}]$ \\
\hline 0.090 & -0.00599 & 0.00188 & 0.049 & 0.065 & 0.041 & 1.165 & 0.044 & 18.35 & 0.052 & 580.0 \\
\hline
\end{tabular}

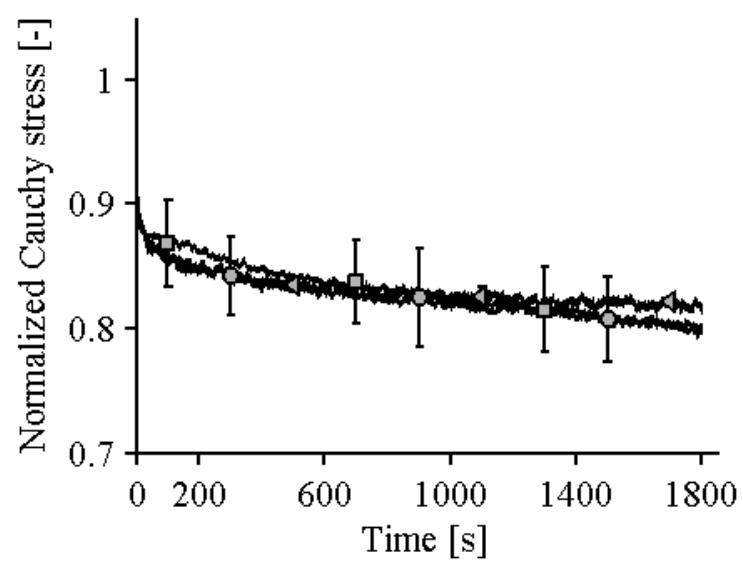

(a)

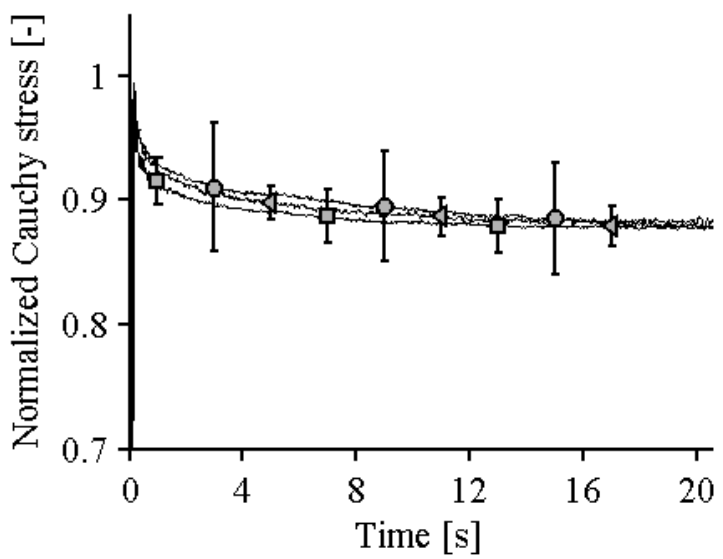

(b)

Figure 8 Normalized Cauchy stress in relaxation experiments a) for the whole time range b) detailed plot up to $20 \mathrm{~s}$, (square symbols $100 \%$ strain, circles $75 \%$ and triangles $50 \%$, error bars indicate the observed standard deviation) 


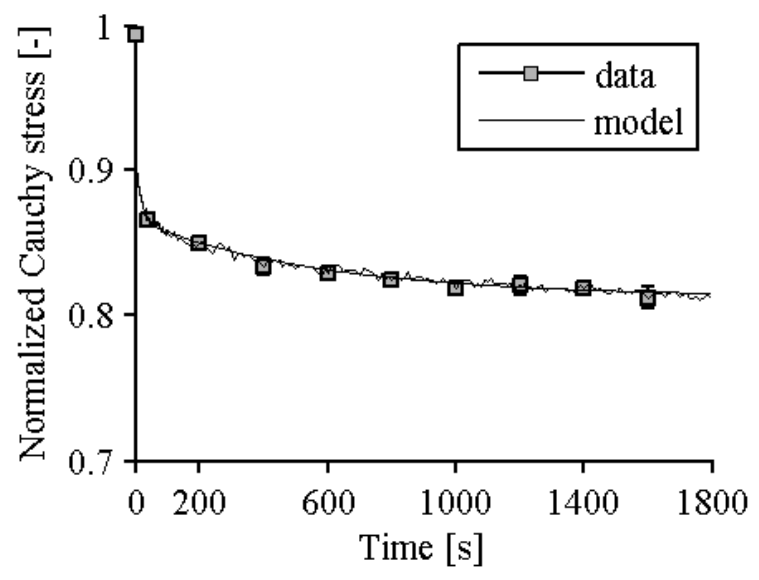

(a)

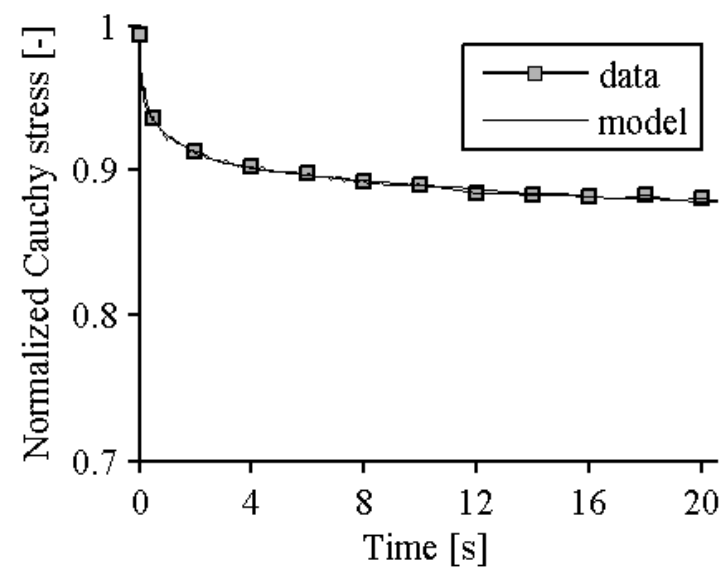

(b)

Figure 9 Comparison of model predictions and average of normalized Cauchy stress a) for the whole time range b) detailed plot up to $20 \mathrm{~s}$

\subsection{MODEL VERIFICATION}

To verify the determined hyper-viscoelastic material model, data of cyclic tensile tests as well as isotonic activation experiments are compared with the respective model predictions. For the cyclic tensile tests a FE model was created using ABAQUS 6.9-1. With the help of a kinematic boundary conditions the model was constrained to $\lambda_{2}=1$ and the measured displacement was applied to the model to calculate stress values (direction 1). For the first cycles of each frequency a comparison of model and experimental data is shown in Figure 10. As can be seen the model well predicts the experimental data. Deviations are less than $5 \%$ for a frequency of $1 \mathrm{~Hz}$ and less than $3 \%$ for a frequency of $5 \mathrm{~Hz}$.

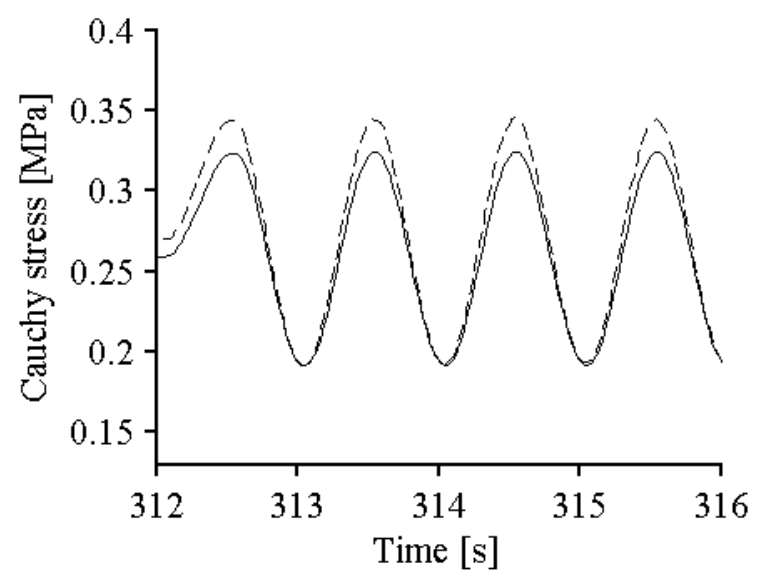

(a)

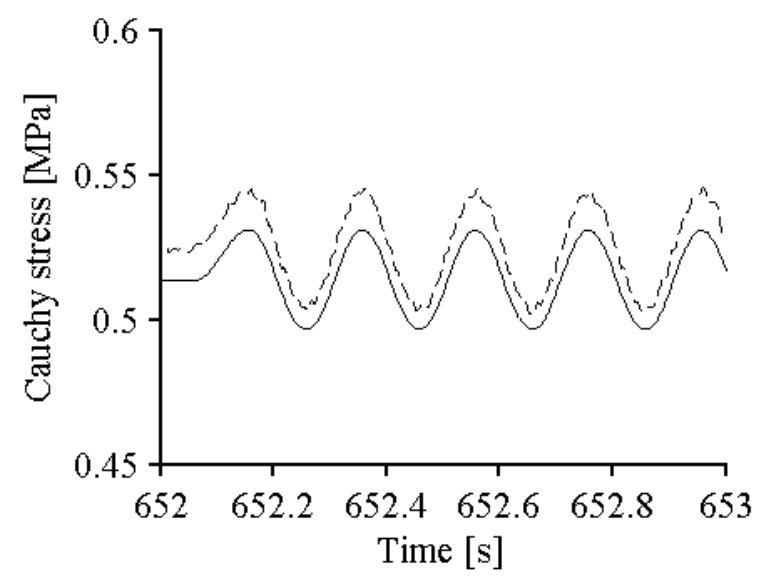

(b)

Figure 10 Model predictions (line) and experimental data (dashed line) of Cauchy stress in cyclic tensile tests (a) for a frequency of $1 \mathrm{~Hz}$ and a strain amplitude of $\pm 10 \%$ (b) for a frequency of $5 \mathrm{~Hz}$ and a strain amplitude of $\pm 2 \%$ 
For activation experiments the following assumptions are made: i) the electric field vector is aligned with the direction 3 (Figure 1) and ii) the value of electric field can be calculated by dividing the applied voltage with the thickness $T$ (Figure 1) of the membrane. Both assumptions neglect that the electric field distribution is inhomogeneous due to the micro structured electrodes. To visualize this effect we calculate the electric field distribution using an electrostatic model in COMSOL. In the model a constant and homogeneous voltage of $1 \mathrm{~V}$ was applied to the micro structured electrodes. The silicone is assumed to follow an ideal dielectric behavior with a relative dielectric permittivity of $2.6^{13}$. As can be seen in Figure 11 the value of the electric field component in thickness direction is not constant but the inhomogeneity vanishes outside a region about the size of the corrugation pattern. The influence of this effect is obviously dependent on the specific corrugation shape and thus on the deformation that is applied to the membrane. In the case of the PolyPower DEAP, the depth of the corrugation is much smaller as compared to the thickness of the membrane. Therefore a homogeneous electric field distribution results in the majority of the material. The value of electric field (component 3 ) that is acting on the majority of material is around $11 \mathrm{~V} / \mathrm{mm}$ and thus very close to the value calculated by dividing the voltage by the inner thickness of $0.09 \mathrm{~mm}$, i.e. $11.11 \mathrm{~V} / \mathrm{mm}$.

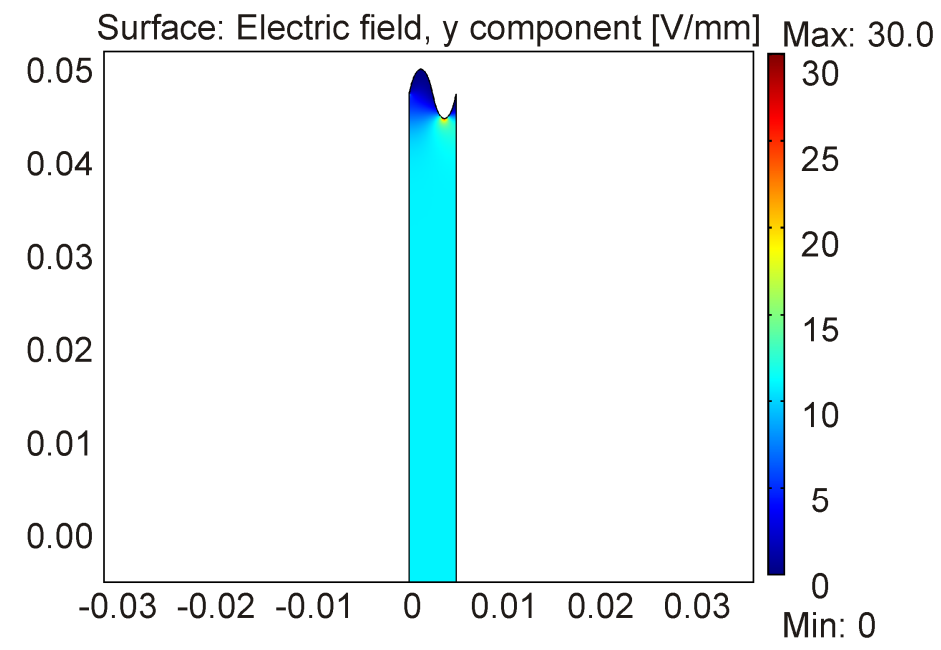

Figure 11 Electric field distribution (component in direction 3) in the micro structured dielectric (color in digital version) 
Further the electrical resistance of the electrodes is assumed to equal zero while the resistance of the dielectric is infinite. This "electro-static" approach is based on the assumption, that the timedependence in mechanical properties is much more pronounced as compared with the time dependence of the electrical properties. For modeling of dynamic applications this approach might not be adequate in all cases.

If the dielectric is subjected to an electric field in direction 3 the electric field causes an additional deformation for a given stress state or reduces the stress state at a given deformation. Thus for an ideal dielectric the long-term stress state can be calculated by subtracting the Maxwell stress tensor from the right hand side of eq.(4):

$$
\underline{\sigma}=2 \underline{F} \frac{\partial W}{\partial I_{1}} \underline{I}^{T}-p-\frac{1}{2} \varepsilon_{0} \varepsilon_{r} E^{2}\left[\begin{array}{ccc}
1 & 0 & 0 \\
0 & 1 & 0 \\
0 & 0 & -1
\end{array}\right]
$$

For a detailed analysis of electromechanical modeling see ${ }^{21}$.

Figure 12 (a) shows model predictions according to eq. (12) using long-term material parameters. Considering the variability typically observed in actuator experiments, the model appears to well represent the behavior of the structure. Since the deformations due to activation are rather small (see Figure 6), Figure 12 (b) and (c) show the ratio of stretch with and without activation. As can be seen the model overestimates this so called "active stretch" for large deformations close to the deformation limit of the corrugated electrodes (forces above $6 \mathrm{~N}$ ).

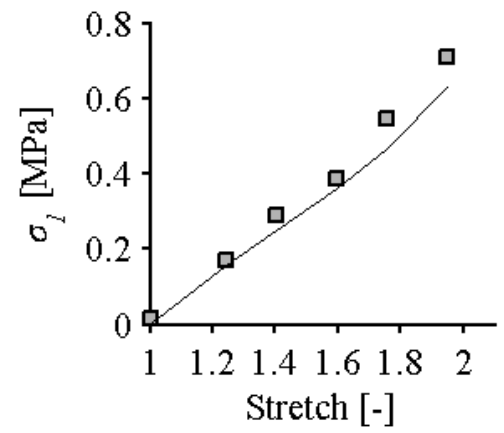

(a)

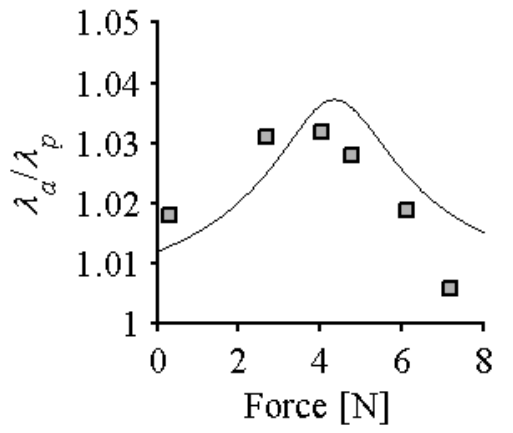

(b)

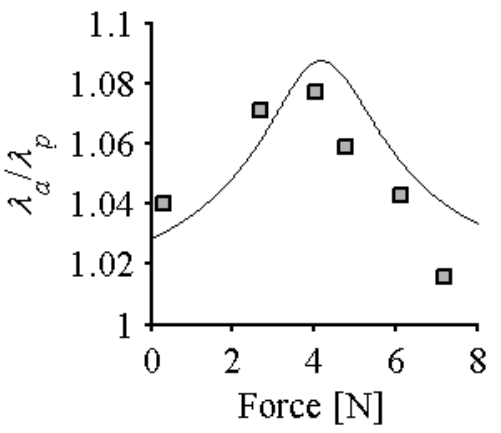

(c)

Figure 12 Comparison of experimental data and model predictions for isotonic tests, a) Cauchy stress vs. stretch in passive tests, b) ratio of stretch at 2 and at $0 \mathrm{kV}, \mathrm{c}$ ) ratio of stretch at 3 and $0 \mathrm{kV}$,

\section{Conclusions}

A large number of passive mechanical tests were conducted to characterize the mechanical behavior of a dielectric membrane with corrugated silver electrodes. The structure was found to possess a nonlinear elastic and time-dependent behavior. Further the structure reveals a pronounced anisotropy 
leading to large differences in mechanical stiffness in the two in-plane directions. We use this property to derive a simplified analytical model for the behavior that holds for both active and passive experiments. Model parameters are provided for the selected model formulation and are shown to predict the structural behavior in passive tensile and isotonic activation tests. We further characterized the time-dependence of mechanical behavior with relaxation experiments. The viscoelastic relaxation leads to a reduction in stress in the order of $20 \%$. We determined Pronyseries parameters that well predict the experimental data and can be used to model the time- or frequency dependent materials response with standard FE software. The model parameters were verified with cyclic tensile tests for different frequencies (up to $5 \mathrm{~Hz}$ ) and different strains.

\section{Acknowledgment}

Financial support from the Swiss National Science Foundation (Project Nr. 200021-107661) is gratefully acknowledged.

\section{REFERENCES}

[1] Pelrine, R., Kornbluh, R., Pei, Q., Stanford, S., Oh, S., Eckerle, J., Full, R., Rosenthal, M., Meijer, K., "Dielectric Elastomer Artificial Muscle Actuators: Toward Biomimetic Motion," Proc. SPIE 4695, 126-137 (2002).

[2] Carpi, F., De Rossi, D., "Dielectric Elastomer cylindrical actuators: electromechanical modeling and experimental evaluation," Mat. Sci. Eng. C, 24, 555-562 (2004).

[3] Schlaak, H. F., Jungmann, M., Matysek, M., Lotz, P., "Novel Multilayer Electrostatic Solid-State Actuators with Elastic Dielectric," Proc. SPIE 5759, 121-133 (2005).

[4] Kovacs, G., Düring, L., Michel, S., Terrasi, G., "Stacked dielectric elastomer actuator for tensile force transmission," Sens. Actuators A, 155, 299-307 (2009).

[5] Anderson, I.A., Hale, T., Gisby, T., Inamura, T., McKay, T., O’Brien, B., Walbran, S., Calius, E.P., “A thin membrane artificial muscle rotary motor," Appl. Phys. A, 98, 75-83 (2009).

[6] Pelrine, R., Kornbluh, R., Eckerle, J., Jeuck, P., Oh, S., Pei, Q., Stanford, S., "Dielectric Elastomers: Generator Mode Fundamentals and Applications, “ Proc. SPIE 4329, 148-156 (2001).

[7] Chiba, S., Waki, M., Kornbluh, R., Pelrine, R., "Innovative Power Generators for Energy Harvesting Using Electroactive Polymer Artificial Muscles," Proc. SPIE 692725, 1-9 (2008).

[8] Koh, S. J. A., Zhao, X., Suo, Z., "Maximal energy that can be converted by a dielectric elastomer generator," Applied Phys. Letters 94, 262902 (2009).

[9] Graf, C., Maas, J., Schapeler, D., "Electromechanical Energy Conversion Using Dielectric Elastomer Generators," Proc. ACTUATOR, 834-837 (2010).

[10] http://www.polypower.com/

[11] Benslimane, M. Y., Kiil, H.-E., Tryson, M. J., "Electro-mechanical properties of novel large strain PolyPower film and laminate components for DEAP actuator and sensor applications," Proc. SPIE 764231, 1-11 (2010).

[12] Benslimane, M. Y., Kiil, H.-E., Tryson, M. J., "Dielectric electro-active polymer push actuators: performance and challenges," Polym. Int., 59, 415-421 (2010).

[13] Jordi, C., Schmidt, A., Kovacs, G., Ermanni, P., „Performance Evaluation of cutting-edge dielectric elastomer for large scale actuator applications," submitted

[14] Wissler, M., Mazza E., "Mechanical behavior of an acrylic elastomer used in dielectric elastomer actuators," Sens. Actuators A, 134, 494-504 (2007).

[15] Schmidt, A., Bergamini, A., Kovacs, G., Mazza, E., "Multiaxial mechanical characterization of Interpenetrating Polymer Network Reinforced Acrylic Elastomer," accepted for publication in Exp. Mech.

[16] Marckmann, G., Verron, E., "Comparison of hyperelastic models for rubber-like materials," Rubber Chem. Technol., 79, 5, 835-858 (2006). 
[17] Yeoh, O. H., "Some Forms of the Strain Energy Function for Rubber," Rubber Chem. Technol., 66, 754-771, (1993).

[18] Holzapfel, G. A., [Nonlinear Solid Mechanics], John Wiley\&Sons, Chichester, 222-226 (2007).

[19] ABAQUS/Standard Manual, Version 6.9-1.

[20] Sarban, R., Mace, B.R., Rustighi, E., Jones, R.W., "Dielectric Electro-Active Polymers in Active Vibration Isolation," Proc. ICAST 9146, (2009).

[21] Suo, Z., "Theory of Dielectric Elastomers," Acta Mechanica Solida Sinica, 23, 6, 549-578 (2010). 